Western Washington University Western CEDAR

\title{
The Morphostatic Limit for a Model of Skeletal Pattern Formation in the Vertebrate Limb
}

\author{
Mark Alber \\ University of Notre Dame \\ Tilmann Glimm \\ Western Washington University, tilmann.glimm@wwu.edu \\ H. George E. Hentschel \\ Emory University \\ Bogdan Kazmierczak \\ Polska Akademia Nauk \\ Yong-Tao Zhang \\ University of Notre Dame \\ See next page for additional authors
}

Follow this and additional works at: https://cedar.wwu.edu/math_facpubs

Part of the Mathematics Commons

\section{Recommended Citation}

Alber, Mark; Glimm, Tilmann; Hentschel, H. George E.; Kazmierczak, Bogdan; Zhang, Yong-Tao; Zhu, Jianfeng; and Newman, Stuart (Stuart A.), "The Morphostatic Limit for a Model of Skeletal Pattern Formation in the Vertebrate Limb" (2008). Mathematics. 56.

https://cedar.wwu.edu/math_facpubs/56 
Authors

Mark Alber, Tilmann Glimm, H. George E. Hentschel, Bogdan Kazmierczak, Yong-Tao Zhang, Jianfeng Zhu, and Stuart (Stuart A.) Newman 


\title{
The morphostatic limit for a model of skeletal pattern formation in the vertebrate limb
}

\author{
Mark Alber \\ Department of Mathematics, University of Notre Dame, Notre Dame, IN 46556-4618 \\ malber@nd.edu \\ Tilmann Glimm \\ Department of Mathematics, Western Washington University, Bellingham, WA 98225 \\ glimmt@wwu.edu \\ H.G.E. Hentschel \\ Department of Physics, Emory University, Atlanta, GA 30322-2430 \\ phshgeh@physics.emory.edu \\ Bogdan Kazmierczak \\ Polish Academy of Sciences, Institute of Fundamental Technological Research, \\ 00-049 Warszawa, Poland \\ bkazmie1@nd.edu \\ Yong-Tao Zhang \\ Department of Mathematics, University of Notre Dame, Notre Dame, IN 46556-4618 \\ yzhang10@nd.edu \\ Jianfeng Zhu \\ Department of Mathematics, University of Notre Dame, Notre Dame, IN 46556-4618 \\ jzhu@nd.edu \\ Stuart A. Newman \\ Department of Cell Biology and Anatomy, Basic Science Building \\ New York Medical College, Valhalla, NY 10595 \\ newman@nymc.edu
}

June 6, 2007 


\begin{abstract}
A recently proposed mathematical model of a "core" set of cellular and molecular interactions present in the developing vertebrate limb was shown to exhibit patternforming instabilities and limb skeleton-like patterns under certain restrictive conditions, suggesting that it may authentically represent the underlying embryonic process (Hentschel et al., 2004). The model, an eight-equation system of partial differential equations, incorporates the behavior of mesenchymal cells as "reactors," both participating in the generation of morphogen patterns and changing their state and position in response to them. The full system, which has smooth solutions that exist globally in time, is nonetheless highly complex and difficult to handle analytically or numerically. According to a recent classification of developmental mechanisms (Salazar-Ciudad et al., 2003), the limb model of Hentschel et al. (2004) is "morphodynamic," since differentiation of new cell types occurs simultaneously with cell rearrangement. This contrasts with "morphostatic" mechanisms, in which cell identity becomes established independently of cell rearrangement. Under the hypothesis that development of some vertebrate limbs employs the core mechanism in a morphostatic fashion, we derive in an analytically rigorous fashion a pair of equations representing the spatiotemporal evolution of the morphogen fields under the assumption that cell differentiation relaxes faster than the evolution of the overall cell density (i.e., the morphostatic limit of the full system). This simple reaction-diffusion system is unique in having been derived in an analytically rigorous fashion from a substantially more complex system involving multiple morphogens, extracellular matrix deposition, haptotaxis, and cell translocation. We identify regions in the parameter space of the reduced system where Turing-type pattern formation is possible, which we refer to as its "Turing space." Obtained values of the parameters are used in numerical simulations of the reduced system, using a new Galerkin finite element method, in tissue domains with nonstandard geometry. The reduced system exhibits patterns of spots and stripes like those seen in developing limbs, indicating its potential utility in hybrid continuum-discrete stochastic modeling of limb development. Lastly, we discuss the possible role in limb evolution of selection for increasingly morphostatic developmental mechanisms.
\end{abstract}

\title{
1 Introduction
}

The growth of experimentally-based knowledge of cell and gene function during embryonic development has enabled increasingly realistic mathematical and computational simulations of cellular pattern formation in multicellular organisms (reviewed in Forgacs and Newman, 2005). Skeletal patterning in the vertebrate limb, i.e., the spatiotemporal regulation of cartilage differentiation ("chondrogenesis") during embryogenesis and regeneration, is one of the best studied examples of such developmental processes (Tickle, 2003; Endo et al., 2004; Brockes and Kumar, 2005; Newman and Müller, 2005). Limb morphogenesis involves subcellular, cellular and supracellular components that interact in a reliable fashion to produce functional skeletal structures. Since these components and in- 
teractions are also typical of other embryonic processes, understanding this phenomenon can provide insights into a variety of morphogenetic events in early development.

The basic organization of the limb bud and adult limb skeleton are similar among the vertebrates (Hinchliffe, 2002). Despite this general conservation, there is extensive morphological and functional diversity arising from variations in the way the cellular-molecular mechanisms that sculpt the limb are employed. Classical observations and experimental studies have demonstrated that the limb skeleton of all vertebrates arises from the loosely packed interior cells of the limb bud ("mesenchyme"). Cartilage, the initially forming skeletal tissue, is replaced by bone later in embryogenesis in species with bony skeletons. The limb skeleton of most vertebrate groups (e.g., birds and mammals) develops in a proximodistal fashion (i.e., the structures closer to the attachment point on the body arising earliest and the successively more distant ones in temporal order). Its elongation and progressive distalization is entirely dependent on a narrow rim of raised cells of the ectoderm, or embryonic skin, running along the distal tip of the paddle-shaped limb bud, the Apical Ectodermal Ridge (AER). The AER also keeps the mesenchymal cells within approximately $0.3 \mathrm{~mm}$ of it in an undifferentiated state; chondrogenesis is preceded by, and dependent on, the transient formation of tight aggregates of cells - "precartilage condensations" - that form at specific sites within the mesenchyme sufficiently distant from the AER (reviewed in Newman, 1988). Urodele (i.e., tail-bearing) amphibians (salamanders and newts) represent an exception to these generalizations in that they lack an AER and the development of their skeletal elements is not strictly proximodistal (Franssen et al., 2005). They are also the only vertebrate species to fully regenerated amputated limbs (Brockes and Kumar, 2005).

Given the complexity of the cell-molecular interactions involved in generating the limb skeleton, theoretical approaches that focus on the commonalities of the developmental process, rather than the element- or limb-type specificities, provide a logical starting point. Under this strategy, the fundamental problem to be addressed by a mathematical model of limb development is accounting for the formation of the generic pattern during skeletogenesis, more specifically, the sequence of transitions from a single bar of cartilage (i.e., the developing humerus or femur) to the two bars of cartilage of the mid-arm or leg, to the rows of nodules constituting the wrist or ankle and the multiple bars of the hand or foot (see Fig. 1). Several models of this process have been based on Turing-type instabilities in reaction-diffusion systems (Newman and Frisch, 1979; Hentschel et al., 2004; Alber et al., 2005a; Cickovski et al., 2005; Miura et al., 2006). In these models the spatiotemporal evolution of various morphogens (i.e., secreted, diffusible gene products) and the cells that respond to them by changing their position and differentiating into cartilage, generates the classic pattern of skeletal elements (reviewed in Newman and Müller, 2005). The most detailed model for vertebrate limb development presented thus far is that of Hentschel et al., 2004. That system of eight partial differential equations (PDEs) was constructed 
largely on the basis of experimentally determined cellular-molecular interactions occurring in the avian and mouse limb bud. The full system has smooth solutions that exist globally in time (Alber et al., 2005a) but is difficult to handle analytically or numerically. By analytically implementing the assumption (proposed to apply to limb development in some vertebrate species; see Discussion) that cell differentiation relaxes faster than the evolution of the overall cell density, we show here that a pair of PDEs can be extracted from the eight-equation system governing the interaction of two of the key morphogens: the activator and the activator-dependent inhibitor of precartilage condensation formation. According to a recent classification of developmental mechanisms (Salazar-Ciudad et al., 2003), the limb model of Hentschel et al. (2004) is "morphodynamic," since differentiation of new cell types occurs simultaneously with cell rearrangement. This contrasts with "morphostatic" mechanisms, in which cell identity becomes established independently of cell rearrangement. Our result, therefore, is a simple but biologically motivated, system that describes the behavior of the pattern-forming limb morphogens in the "morphostatic limit."

In the simplified two-equation system for morphogen evolution, determining parameter ranges under which the system can give rise to patterns (what we refer to as the "Turing space" of the system) is much more tractable than for the full morphodynamic system. The reduced reaction-diffusion system can also feasibly be used in a variety of computational models in which additional morphogens, responsive model cells (specified to behave according to the assumptions under which the morphogen subsystem was isolated) and realistic limb bud geometries, can be introduced. The fact that the reduced equations are derived analytically from the morphodynamic system makes them biologically better justified then ad hoc reaction kinetics used in earlier hybrid continuum-discrete models of the limb (Chaturvedi et al., 2005; Cickovski et al., 2005). To illustrate the pattern-forming capability of the reduced system in geometrically irregular domains we present simulations under selected parameter ranges using a novel Galerkin finite element method.

The paper is organized as follows: In Section 2 we give a brief overview of the model and summarize the findings of Hentschel et al. (2004) in compact form with emphasis on the biological significance. The main mathematical content of the paper follows in Section 3. There we analyze the morphostatic limit of the full model, that is, the reduced equations we obtain by making certain (biologically motivated) assumptions as indicated above, the most important one being that cell differentiation is a faster process than the evolution of the overall cell density. We then prove a result on the positivity of solutions of the corresponding systems of PDEs. We then consider the fundamental requirement that our system be able to give rise to Turing patterns, and thus identify parameter ranges where a Turing instability is possible. Based on the requirement of the possibility of Turing patterns, we can derive necessary conditions on our system, which translate into testable predictions concerning the behavior of cells. In particular, we determine the choice 
of parameters which guarantees existence of solutions of the system of equations in the morphostatic limit. Such solutions are required to be non-negative and to not approach infinite values (i.e., blow up) in finite time. Obtained values of the parameters are used in numerical simulations of the system on irregular tissue domains (section 4). In section 5 we discuss the implications of the pattern formation mechanisms in the morphostatic limit for development and evolution of the limb.

\section{Modeling limb skeletal pattern formation}

\section{$2.1 \quad$ Biological background}

In the last two decades the molecules that mediate many key processes in limb morphogenesis and pattern formation have been identified:: the AER is a source of, and can be replaced by, a set of secreted, diffusible fibroblast growth factors (FGF-2, FGF-8) (Martin, 1998); cell condensation is mediated by a secreted extracellular matrix protein, fibronectin (Frenz et al., 1989; Gehris et al., 1997); fibronectin itself is induced by one or more of a family of secreted, diffusible factors (TGF- $\beta$ s) (Leonard et al., 1991) which also induce their own production (positive autoregulation) (Miura and Shiota, 2000a). Spatial expansion of condensations is limited by a laterally acting inhibitory factor released from condensing cells in response to ectodermal FGFs (Moftah et al., 2002). While the molecule(s) mediating this inhibitory effect has not been identified, the effect appears to be mediated by both FGF receptor 2 (Moftah et al., 2002) and the Notch signaling pathway (Fujimaki et al., 2006). Finally, differential expression of a variety of molecules, including the secreted factor Sonic hedgehog (Shh) and the Hox family of transcription factors, cause skeletal elements to differ from one another in morphological detail within a given limb (Tickle, 2003) and are also thought to be responsible for differences in limb morphology between species (Hinchliffe, 2002).

This set of cell and molecular interactions suggests a model for skeletal pattern formation based on a Turing-type pattern forming mechanism that has undergone evolutionary fine-tuning (see Turing, 1952; see also Newman and Müller, 2005 for a review). A number of caveats are relevant in considering this class of mechanisms, however. While it continues to be a matter of debate whether morphogens are indeed transported through tissues by diffusion (Merkin and Sleeman, 2005; Lander, 2007), recent quantitative studies have provided evidence for diffusion of both TGF- $\beta$-type (Lander et al., 2002; Williams et al., 2004) and FGF-type (Filion and Poper, 2004) morphogens during key developmental processes. In any case, alternative means of transport, such as transcytosis (Entchev et al. 2000) and long-distance signaling by filopodia (De Joussineau et al., 2003), can play the same formal role as diffusion in Turing-type pattern-forming systems (Nijhout, 2003). Another claimed drawback of such models is that, unlike many developmental outcomes, the patterns produced by Turing-type systems are scale-dependent and symmetric. 
Beyond this, the following experimental findings, count in favor of the relevance of a reaction-diffusion mechanism for limb pattern formation: (i) The pattern of precartilage condensations in limb mesenchyme in vitro changes in a fashion consistent with reactiondiffusion mechanism (and not with an alternative mechanochemical mechanism) when the density of the surrounding matrix is varied (Miura and Shiota, 2000b); (ii) exogenous FGF perturbs the kinetics of condensation formation by limb precartilage mesenchymal cells in vitro in a fashion consistent with a role for this factor in regulating inhibitor production in a reaction-diffusion model (Miura and Maini, 2004); (iii) the "thick-thin"" pattern of digits in the Doublefoot mouse mutant can be accounted for by the assumption of that the normal pattern is governed by a reaction-diffusion process the parameters of which are modified by the mutation (Miura et al., 2006); (iv) the scale-dependence of reaction-diffusion systems (i.e., the addition or loss of pattern elements when the tissue primordium has variable size), sometimes considered to count against such mechanisms for developmental processes, may actually represent the biological reality in the developing limb. Experiments show, for example, that the number of digits that arise is sensitive to the anteroposterior (thumb-to-little finger breadth) of the developing limb bud, and will increase (Cooke and Summerbell, 1981) or decrease (Alberch and Gale, 1983) over typical values if the limb is broadened or narrowed.

\subsection{The morphodynamic model}

Our starting point is the system of equations proposed in Hentschel et al. (2004), which describes the cell dynamics and the chemical processes during limb bud formation. It has the following form:

$$
\begin{gathered}
\partial c / \partial t=D \nabla^{2} c-k c+J(x, t) \\
\partial c_{a} / \partial t=D_{a} \nabla^{2} c_{a}-k_{a} c_{i} c_{a}+J_{a}^{1} R_{1}+J_{a}\left(c_{a}\right) R_{2} \\
\partial c_{i} / \partial t=D_{i} \nabla^{2} c_{i}-k_{a} c_{i} c_{a}+J_{i}\left(c_{a}\right) R_{2} \\
\partial R_{1} / \partial t=D_{\text {cell }} \nabla^{2} R_{1}-\chi \nabla \cdot\left(R_{1} \nabla \rho\right)+r R_{1}\left(R_{e q}-R\right)+k_{21} R_{2}-k_{12}\left(c, c_{a}\right) R_{1} \\
\partial R_{2} / \partial t=D_{\text {cell }} \nabla^{2} R_{2}-\chi \nabla \cdot\left(R_{2} \nabla \rho\right)+r R_{2}\left(R_{e q}-R\right)+k_{12} R_{1}-k_{21} R_{2}-k_{22} R_{2} \\
\partial R_{2}^{\prime} / \partial t=D_{\text {cell }} \nabla^{2} R_{2}^{\prime}-\chi \nabla \cdot\left(R_{2}^{\prime} \nabla \rho\right)+r R_{2}^{\prime}\left(R_{e q}-R\right)+k_{22} R_{2}-k_{23} R_{2}^{\prime} \\
\partial R_{3} / \partial t=r_{3} R_{3}\left(R_{3 e q}-R_{3}\right)+k_{23} R_{2}^{\prime} \\
\partial \rho / \partial t=k_{b}\left(R_{1}+R_{2}\right)+k_{b}^{\prime} R_{2}^{\prime}-k_{c} \rho .
\end{gathered}
$$

In the above equations, $c$ denotes the concentration of FGFs, $c_{a}$ the concentration of TGF- $\beta$ (activator), $c_{i}$ concentration of the inhibitor, $R_{1}, R_{2}, R_{2}^{\prime}, R_{3}$ densities of different kinds of cells and $\rho$ density of fibronectin. $R=R_{1}+R_{2}+R_{2}^{\prime}$ is the overall density of 
the mobile cells. As we are not interested in the growth phenomena of the limb, but only in the processes of the pattern formation, we consider the system's behavior in a smooth (i.e., at least of $C^{2+\nu}$ class) domain $\Omega \subset \mathbb{R}^{n}, n \geq 2$, which is assumed to be fixed in space and time, based on the assumption that $\tau_{m} \ll \tau_{g}, \tau_{d} \ll \tau_{g}$, where $\tau_{m}, \tau_{d}$ and $\tau_{g}$, are the characteristic times of morphogen evolution, cell differentiation, and limb growth, respectively. In terms of the model parameters, $\tau_{m} \sim L^{2} / D$, where $L$ is the characteristic length scale, as above, and $D$ is the morphogen diffusivity, $\tau_{d} \sim 1 / k$, where $k$ is the ratelimiting kinetic term for morphogen production, and $\tau_{g} \sim L / V$, where $V$ is the typical convective time scale for the viscoelastic tissue.

All of the functions are subject to no-flux boundary conditions

$$
\frac{\partial c}{\partial \mathbf{n}}=\frac{\partial c_{a}}{\partial \mathbf{n}}=\frac{\partial c_{i}}{\partial \mathbf{n}}=\frac{\partial \rho}{\partial \mathbf{n}}=\frac{\partial R_{1}}{\partial \mathbf{n}}=\frac{\partial R_{2}}{\partial \mathbf{n}}=\frac{\partial R_{2}^{\prime}}{\partial \mathbf{n}}=\frac{\partial R_{3}}{\partial \mathbf{n}}=0 .
$$

and smooth initial condition at $t=0$. Here $\mathbf{n}=\mathbf{n}(x)$ is the outward normal to $\partial \Omega$ at point $x$. Therefore, cells' and chemicals' fluxes are equal to zeros at the boundary.

This system is difficult to treat analytically for two reasons. First, diffusion constants for $R_{3}$ cells and fibronectin $(\rho)$ are equal to zero. Second, the presence of the terms $\chi \nabla \cdot\left(R^{*} \nabla \rho\right), R^{*}=R_{1}, R_{2}, R_{2}^{\prime}$, may lead to a blow-up in finite time of the solutions. Therefore, we have investigated the system under additional assumptions in (Alber et al., 2005a). In this paper we introduce limiting conditions (see below) which also simplify analysis of the system and and allow us to study behavior of its solutions.

\section{Morphostatic Limit}

In what follows we demonstrate non-negativity of solutions and describe sufficient conditions for the existence of Turing-type pattern solutions of the morphostatic limit of the system described in the previous section. The key assumption is that cell differentiation happens on a faster time scale than the change of cell densities due to individual cell motion, yielding what may be termed the "morphostatic limit" of the full system, with reference to the classification of composite developmental mechanisms by Salazar-Ciudad et al. (2003)

\subsection{Basic assumptions and reduction to two-equation system}

Recall that a reaction-diffusion system may produce spot- or stripe-like patterns in two dimensional domain, and, under appropriate conditions, nodules and bar-like structures in a three-dimensional domain (Alber et al., 2005b). The type of the resulting pattern depends on the geometry of the domain as well as on the initial conditions.

The Turing pattern which evolves from initial conditions is most likely the one which corresponds to the linearly unstable wavelength, i.e., the wavelength $k$ for which the positive eigenvalue of the matrix $\left[\mathbf{A}-k^{2} D\right]$ is maximal (see Murray (1993)). Here A 
denotes the Jacobian matrix of the linearization of the system near its constant steady state, stable with respect to spatially homogeneous perturbations, and $D$ is a diffusion matrix. However, in many developmental models including the one discussed in this paper, the initial conditions are not simply random fluctuations. For example, pattern can be initiated at one end of a spatial domain and then spread from there. In such cases patterns other than the one corresponding to the most unstable wavelength, may emerge due to the nonlinear nature of the system.

We add an assumption to those made in Hentschel et al. (2004)), namely that the overall mobile cell density $R=R_{1}+R_{2}+R_{2}^{\prime}$ is spatially homogeneous and does not depend on time. (See also Chaturvedi et al., 2005 and Cickovski et al., 2005). We are mainly interested in the onset of patterns, before condensation takes place, so that the density of condensing cells $R_{2}^{\prime}$ is effectively zero. Further, since no mobile cells become immobile through differentiating into cartilage cells (since we consider the first differentiation event $\mathrm{R}_{1} \rightarrow \mathrm{R}_{2}$ cells), the overall mobile cell density remains approximately constant over the time scales we are concerned with, provided cell division is a slow process. The mobile cell density is also spatially uniform, since in addition to the lack of condensation in the $R_{1} \rightarrow R_{2}$ transition, cell division rates are uniform to a distance of 0.4-0.5 from the AER (Stark and Searls, 1973) This comprises the $0.3 \mathrm{~mm}$ apical zone within which condensation is blocked by high levels of FGF and all or most of the morphogenetically active zone (Hentschel et al., 2004).

With these simplifications, system (2.1)-(2.8) reduces to following two evolution equations for the morphogens

$$
\begin{aligned}
\partial c_{a} / \partial t & =D_{a} \nabla^{2} c_{a}+U\left(c_{a}\right)-k_{a} c_{a} c_{i} \\
\partial c_{i} / \partial t & =D_{i} \nabla^{2} c_{i}+V\left(c_{a}\right)-k_{a} c_{a} c_{i}
\end{aligned}
$$

with the initial and boundary conditions

$$
\begin{gathered}
c_{a}(x, 0)=c_{a 0}(x), \quad c_{i}(x, 0)=c_{i 0}(x) \\
\frac{\partial c_{a}}{\partial \mathbf{n}}=\frac{\partial c_{i}}{\partial \mathbf{n}}=0 .
\end{gathered}
$$

Here $\mathbf{n}=\mathbf{n}(x)$ is the outward normal to $\partial \Omega$ at point $x$. (We assume that $\Omega \subset \mathbb{R}^{n}, n \geq 1$, is such that its boundary $\partial \Omega$ is of class $C^{2+\nu}, \nu \in(0,1)$.) The functions $U$ and $V$ are given by

$$
U\left(c_{a}\right)=\left[J_{a}^{1} \alpha\left(c, c_{a}\right)+J_{a}\left(c_{a}\right) \beta\left(c, c_{a}\right)\right] R_{e q}, \quad V\left(c_{a}\right)=J_{i}\left(c_{a}\right) \beta\left(c, c_{a}\right) R_{e q} .
$$


In Eqs. (3.13) $J_{a}^{1}$ and $k_{a}$ are assumed to be constant. $J_{a}\left(c_{a}\right)$ and $J_{i}\left(c_{a}\right)$ are taken in the form

$$
\begin{gathered}
J_{a}\left(c_{a}\right)=J_{a \max }\left(c_{a} / s\right)^{n} /\left[1+\left(c_{a} / s\right)^{n}\right], \\
J_{i}\left(c_{a}\right)=J_{\text {imax }}\left(c_{a} / \delta\right)^{q} /\left[1+\left(c_{a} / \delta\right)^{q}\right]
\end{gathered}
$$

with $n \geq 1, q \geq 1$. We further have

$$
\begin{gathered}
\alpha\left(c, c_{a}\right)=1 / Z\left(c, c_{a}\right), \\
\beta\left(c, c_{a}\right)=K_{1}\left(c, c_{a}\right) / Z\left(c, c_{a}\right), \\
\text { with } Z\left(c, c_{a}\right)=1+K_{1}\left(c, c_{a}\right)\left(1+K_{2}\right),
\end{gathered}
$$

where $K_{2}$ is a positive constant, and

$$
K_{1}\left(c, c_{a}\right)=K(c)\left(c_{a} / \widetilde{s}\right) /\left[1+\left(c_{a} / \widetilde{s}\right)\right]
$$

In what follows, we will assume that $c=$ const and $\widetilde{s}=s$ and will use the notation

$$
K\left(c_{a}\right)=K(c)\left(c_{a} / s\right) /\left[1+\left(c_{a} / s\right)\right]=K_{\max }\left(c_{a} / s\right) /\left[1+\left(c_{a} / s\right)\right] .
$$

\subsection{Existence and non-negativity of the solutions}

Here we determine the choice of parameters and initial conditions which would guarantee existence of solutions of the morphostatic limit system of equations described in the previous section. To be biologically plausible they need to be non-negative and not approach infinite values in finite time (blow up in finite time). Obtained values of the parameters will be used in later sections in numerical simulations in tissue domains with nonstandard geometry.

A Turing structure is a spatial pattern which appears close to a spatially homogeneous steady state $\left(c_{a *}, c_{i *}\right)$ that is stable in the absence of diffusion and which loses stability in favor of the Turing pattern in the presence of diffusion.

Therefore, if the constant steady state is positive, then for the Turing pattern we also have that $c_{a}(x)>0, c_{i}(x)>0$. However, it is important to determine whether the condition $c_{a}(x, t) \geq 0, c_{i}(x, t) \geq 0$ holds for all possible solutions of the system (3.9)(3.10). Indeed, a system of equations that allows concentrations to become negative if the initial conditions are nonnegative is clearly non nonphysical and is an unsuitable model for biological development. 
By successive applications of the maximum principle for parabolic operators it can be shown that non-negative initial conditions in the system (3.9)-(3.10) imply that the concentrations remain non-negative for all times. To be more precise, if one supposes that $c_{a}(x, 0) \geq 0, c_{i}(x, 0) \geq 0$, then for any $t>0$ in the maximal interval of existence of the solution this yields that $c_{a}(x, t)>0$ and $c_{i}(x, t) \geq 0$ for $x \in \bar{\Omega}$.

We also established the bounds for the solutions in the form of the invariant rectangles theorem. Suppose that $R_{e q}>0, J_{a}^{1}>0$ and $J_{i, \max }>J_{a, \max }$. Then there exist positive constants $\underline{c}_{i}, \underline{c}_{a}, \bar{c}_{a}>\underline{c}_{a}, \bar{c}_{i}>\underline{c}_{i}$ such that if the initial conditions $\left(c_{a 0}(x), c_{i 0}(x)\right)$ are contained in the rectangle $I:=\left[\underline{c}_{a}, \bar{c}_{a}\right] \times\left[\underline{c}_{i}, \bar{c}_{i}\right]$, then there exists a unique solution $c_{a}(x, t), c_{i}(x, t)$ of the initial-boundary value problem (3.9)-(3.10) (satisfying conditions (3.11) and (3.12)) with values contained in the rectangle $I$.

$$
\begin{aligned}
\bar{c}_{i} & =\sup _{c_{a} \geq 0} \frac{V\left(c_{a}\right)}{k_{a} c_{a}} \\
\underline{c}_{a} & =\inf _{c_{a} \geq 0} \frac{U\left(c_{a}\right)}{k_{a} \bar{c}_{i}} \\
\bar{c}_{a} & =\sup \left\{\tilde{c}_{a} \mid \frac{U\left(\tilde{c}_{a}\right)}{\tilde{c}_{a}} \geq \min _{\underline{c}_{a} \leq c_{a} \leq \tilde{c}_{a}}\left[\frac{V\left(c_{a}\right)}{c_{a}}\right]\right\} \\
\underline{c}_{i} & =\inf _{\underline{c}_{a} \leq c_{a} \leq \bar{c}_{a}} \frac{V\left(c_{a}\right)}{k_{a} c_{a}} .
\end{aligned}
$$

Thus, the concentrations are always bounded and positive if the initial conditions lie between appropriate levels. Biologically, this means that the activator and inhibitor concentrations always remain above a certain threshold and that there is no blow-up of concentrations. The proof of the above existence statements, modulo some additional considerations, follows from the sub- and super solution method (e.g. Pao (1992)). As the proof is rather straightforward we have left the details to the reader.

Finally, it follows from the definition of the constants $\underline{c}_{a}, \bar{c}_{a}, \underline{c}_{i}$ and $\bar{c}_{i}$ that any nonnegative spatially homogeneous steady state $\left(c_{a}^{*}, c_{i}^{*}\right)$ of the system must lie in the square $S=\left[\underline{c}_{a}, \bar{c}_{a}\right] \times\left[\underline{c}_{i}, \bar{c}_{i}\right]$. In particular, for $J_{i, \max }>J_{a, \max }$ a solution bifurcating from a positive stable constant steady state via the Turing bifurcation stays positive and its values lie in $S$.

\subsection{Potential for pattern formation}

One of our goals here is to find possible sets of model parameters for which Turing bifurcation can take place. In Appendix A we derive general conditions determining Turing bifurcation for the system considered in this paper. These conditions are characterized in terms of parameters $\delta$ and $s$, which appear in the activator- and inhibitor production 
rate functions $J_{a}$ and $J_{i}$, respectively. These functions have Michaelis-Menten form. The constants $s$ and $\delta$ denote the concentrations which separate the linear response phase from the saturation response phase. Our main result is that the Turing instability occurs only when the ratio $\delta / s$ is of the order of 1 . This is illustrated by a numerical example in Fig. 2. The biological significance of our findings is discussed in the Discussion (Section 5).

In what follows we consider a number of different biologically interesting cases and determine whether the system can give rise to Turing patterns in each cases.

To characterize these cases we use the following ratios of the parameters

$$
z=c_{a} / s, \quad r=J_{a}^{1} /\left(K_{1 \max } J_{\text {amax }}\right), \quad w=J_{\text {imax }} / J_{\text {amax }} .
$$

Here $J_{a}^{1}$ is assumed to be small compared to $J_{\text {amax }}$ (this is a biologically motivated assumption, see Hentschel et al., 2004). Hence we assume that $r \ll 1$.

Let $K_{2}>0, K_{\max }, s \in \mathbb{R}$ and $\mathbb{R} \ni n \geq 1$ be given constants. Let $K, J_{a}$ and $J_{i}$ be given by (3.18),(3.14) and (3.15) respectively with $q=n$; that is,

$$
\begin{aligned}
& J_{a}\left(c_{a}\right)=J_{\text {amax }} \frac{\left(c_{a} / s\right)^{n}}{1+\left(c_{a} / s\right)^{n}} \\
& J_{i}\left(c_{a}\right)=J_{\text {imax }} \frac{\left(c_{a} / \delta\right)^{n}}{1+\left(c_{a} / \delta\right)^{n}} .
\end{aligned}
$$

We obtained the following results for the system (3.9)-(3.10):

(i) Case $1: \delta / s \ll 1$. Suppose $q=n=1$. Then for any $r=J_{a}^{1} /\left(K_{1 \max } J_{\text {amax }}\right)>0$, there exists $\delta_{0}=\delta_{0}(r)>0$ such that the system does not satisfy the conditions for the existence of Turing instability at any positive spatially homogeneous steady state if $\delta<\delta_{0}$.

(ii) Case 2: $\delta / s=1$. Suppose $\delta=s$. Then there exists a nonempty set of parameters $w=J_{\text {imax }} / J_{\text {amax }}$ with $w>1$ and small enough $r=J_{a}^{1} /\left(J_{\text {amax }} K_{\max }\right)$ such that at the (unique) positive steady state the conditions for the existence of the Turing instability are satisfied.

(iii) Case 3: $\delta / s \gg 1$. Suppose $q=n=1$. Assume further that $w=J_{\text {imax }} / J_{\text {amax }}>1$. Then there exists an $a_{0}>0$ and $r_{0}>0$ with the following property. If $\delta>0$ is such that $a=s / \delta<a_{0}$ and $J_{a}^{1}>0$ is such that $r=J_{a}^{1} /\left(K_{1 \max } J_{a \max }\right)<r_{0}$, then the system does not have Turing instability at any positive spatially constant steady state.

The proof of these statements is presented in Appendix B. 


\subsection{The Turing space for the morphostatic system}

In Fig. 3A, where $\delta / s$ is plotted against Jimin/Jimax with other parameters held constant (see Figure legend for the values), the shaded area represents those points in parameter space where a Turing bifurcation is possible. The graph shows that such a bifurcation cannot occur at the positive steady state for small $\delta / s$ and for large $\delta / s$. Furthermore, for $J_{\text {imin }}<J_{\text {amax }}$ a Turing bifurcation is also not possible.

In Fig. $3 \mathrm{~B} \delta / s$ is plotted against $R_{e q}$ with values of other parameters listed in the figure legend. This graph shows that a Turing bifurcation is only possible if $\delta / s$ is close to 1 . It also indicates that a Turing bifurcation is not possible if the cell density $R_{e q}$ is too high. In fact, for increasing $R_{e q}$, the spatially homogeneous steady state eventually becomes unstable.

\section{Morphogen Dynamics in an Irregular Domain}

In addition to initial conditions and choice of parameters, patterns in reaction-diffusion systems depend sensitively on domain size and shape (Lyons and Harrison, 1992; Crampin et al., 2002; Zykov and Engel, 2004). Since the natural shape of a limb bud (Fig. 1), and its subdomains such as the active zone have non-standard geometries, we developed a mathematical formalism based on the finite element methods (Johnson, 1987), to handle the complicated geometries and solve morphostatic reaction-diffusion system (3.9-3.10) numerically. Our formalism belongs to the Discontinuous Galerkin finite element (DGFE) methods, which use completely discontinuous piecewise polynomial space for the numerical solution and the test functions. Major advances in the development of DGFE methods were presented in a series of papers by Cockburn et al. (1989a; 1989b; 1990; 1991; 1998a; 1998b).

The flexibility and efficiency of DGFE methods make them attractive for biological applications. Recently, Cheng and Shu (2006) developed a new DGFE method for solving time dependent PDEs with higher order spatial derivatives. The scheme is formulated by repeated integration by parts of the original equation and careful treatment of the discontinuity of the numerical solutions on the interface of the neighboring elements, which is important for the stability of the DGFE methods. It is easier to formulate and implement and requires less storage and CPU cost than the usual DGFE methods for PDEs with higher order spatial derivatives. We adopted the discontinuous Galerkin finite element numerical approaches of Cheng and Shu (2006) and implemented it on both 2D rectangular and triangular meshes to solve the reaction-diffusion system (3.9)-(3.10).

Patterns in reaction-diffusion systems are sensitive to the domain size and geometrical shape. The shape of the developing limb bud undergoes continuous changes. The DGFE approach can handle the irregular shapes easily by using triangular meshes to fit the domain. Both spot-like and stripe-like patterns are observed in simulations of the steady 
state solution of the reaction-diffusion system (3.9)-(3.10) on domains with different sizes. To simulate the pattern of realistic shapes of morphogenetically active zones in the limb bud, we randomly perturbed the rectangular boundary of the active zone, and used triangular mesh to fit the irregular boundary. In Fig.4A, the triangular meshes are indicated to show the fit of computational mesh to the irregular boundary. The vertical length of the domain is roughly 0.65 , and the horizontal length is 0.15 arbitrary units. A flood contour plot of the steady-state of the concentration of the activator $c_{a}$ is shown. Different colors in the domain represent different values of $c_{a}$.

Simulations using the domain aspect ratio of Fig.3A exhibited stripe-like patterns. To examine the pattern dependence on the ratio of horizontal length and vertical length of the active zone, we fixed the vertical length to be 1 , but changed the horizontal length successively. The steady state patterns are shown in Fig.4B, and we can observe the changing of stripe-like patterns (i.e., analogous to long bones and digits) to spot-like patterns (i.e., analogous to wrist and ankle bones) when the horizontal length is increased. This result serves as a proof-of-principle that the morphostatic system for morphogen dynamics exhibits tissue-domain shape-dependence of pattern formation, as required in models for limb morphogenesis in this general framework (e.g., Newman et al., 1979; Hentschel et al., 2004).

\section{Discussion}

In this paper we have investigated a mathematical model for the generation of cartilaginous primordia of the vertebrate limb skeleton (Hentschel et al., 2004) under certain restrictive assumptions, which correspond to the "morphostatic" limit according to the classification by Salazar-Ciudad et al. (2003). Although the full model includes extracellular matrix deposition and cell rearrangement via haptotaxis, the reduced model only describes the dynamics of the activating and inhibitory morphogens that control the initiation of chondrogenesis. We were mainly interested in the parameter ranges for which the appearance of (chemical) Turing patterns is possible, thereby breaking the symmetry of the spatially homogeneous steady state. The question of the (temporal) stability of these patterns and how these chemical patterns give rise to, and then interact with, spatial patterns in the cell densities were not dealt with; however, certain hypotheses on the role of the extracellular matrix molecule fibronectin in stabilizing spatial patterns have been put forward in Hentschel et al. (2004) and Alber et al. (2005b).

We assumed that cell differentiation is faster than the changes in the overall cell density, following the arguments in Hentschel et al. (2004). An additional assumption was that the spatial variations in the densities of the various cell types involved are small and can be replaced by a constant density for the analysis of the evolution of the morphogen concentrations. Note that in this case, no a priori assumption regarding the relative 
magnitudes of the time scales for the evolution of the morphogen concentration and the cell densities is made. We were able to find a broad set of parameters for which the necessary conditions for the Turing instability are fulfilled. Our analysis considers a broad class of models characterized by different coefficients in Michaelis-Menten kinetics. Our results suggest that the precise choice of these coefficients does not influence the possibility of the Turing instability. In this sense our considerations are robust. We also proved a non-negativity result which asserts that the activator and inhibitor-concentrations cannot vanish in our model.

For our system to exhibit a Turing-type instability in the morphostatic limit several constraints on morphogen dynamics must be met. In particular, our results indicate the following qualitative predictions:

1) The maximum production rate of the inhibitor by $R_{2}$ cells (i.e., cells bearing FGF receptor 2) exceeds their rate of production of the activator TGF- $\beta$.

2) The threshold levels of local TGF- $\beta$ concentration that elicit maximal production rates by $\mathrm{R}_{2}$ cells of TGF- $\beta$, and of inhibitor, must be of roughly the same order of magnitude.

Knowledge of the cellular biochemistry and the resolution of current techniques do not permit the relevant measurements to be made either in the limb bud or even in micromass cultures at present. These predictions, however, are testable consequences of our assumptions, both in in vivo and in vitro living systems (at least in principle), and in silico, where the biologically and analytically authentic morphogen system represented by (2.10)-(2.11) can be employed in hybrid discrete-continuum simulations in lieu of the ad hoc morphogen systems used previously (Izaguirre et al., 2004; Chaturvedi et al., 2005; Cickovski et al., 2005).

The morphostatic limit for the system (2.1)-(2.8) was introduced to make identification of the Turing space for this system analytically tractable, but it may also represent a reality of development for most or some tetrapod embryos. The establishment of the limb skeletal pattern in chicken embryos occurs over 4 days, while the same process in human embryos occurs over 4 weeks. Since the spatial scales of limb pattern formation in the two species are similar, one or more of the dynamical processes involved - morphogen evolution, cell differentiation, cell mobility - must differ substantially between the different species. This strongly suggests that the parameters we have considered here and in Hentschel et al. (2004) have been subject to natural selection. Transformation of an inherently morphodynamic system into a morphostatic one by, for example, slowing the rate of cell movement, is a plausible evolutionary scenario for evolutionary changes in limb development and in other systems with similar properties.

What might be the selective advantage of a developmental mechanism achieving a morphostatic status? There is good evidence that the limb's morphology was more inconsistent at earlier stages of its evolution than at present. Ancient tetrapod limbs, for 
example, often exhibited great variability in digit number within the same group, with these numbers sometimes exceeding seven or eight (Coates and Clack, 1990). Significantly, this variable polydactylous condition can be achieved in the mouse by knocking out Shh and certain of its modulators (Litingtung et al., 2002). The atypical form of the limb skeleton of Tiktaalik, the recently discovered transitional form between lobefinned fish and tetrapods, suggests that at earlier evolutionary stages limbs were even less constrained (Daeschler et al., 2006; Shubin et al., 2006). Eventually, however, the limb settled into a stereotypical plan, typically pentadactylous (five fingers and toes), but even when not, stable within phylogenetic groups (Hinchliffe, 2002). This suggests that an effect of evolution was to stabilize generation of a standard phenotype, a phenomenon known as developmental canalization (Waddington, 1942). Salazar-Ciudad and coworkers (Salazar-Ciudad et al. 2003; Salazar-Ciudad and Jernvall, 2005; Salazar-Ciudad, 2006) have proposed that morphodynamic mechanisms, in which change in cell state and cell rearrangement occur simultaneously, are more prolific morphologically (i.e., "evolvable") when their constituent genes are mutated than are morphostatic mechanisms, in which these processes occur in a sequential fashion.

Separation of the time-scale of cell movement from that of morphogen pattern dynamics is therefore one strategy by which natural selection can achieve developmental and evolutionary robustness (Wagner, 2005). The question remains, however, of whether reaction-diffusion mechanisms per se, which are inherently sensitive to parameter variation, would persist as important components of developmental processes over long evolutionary periods. For developmental processes in which the detailed morphological outcome is not under strict control, such as branching morphogenesis in the lung (Miura and Shiota, 2002; Hartmann and Miura, 2006) or exocrine glands (Nelson et al., 2006), reaction-diffusion processes are sufficient to generate functional patterns. The functions of the vertebrate limb (running, grasping, swimming, flight) in different species, however, is tied to precise morphologies. The structure of the limb has therefore been under a high degree of selection pressure over the course of evolution. While the limb skeletal pattern may have had it origins in self-organizing reaction-diffusion processes, eventually it would come to be generated by more precise mechanisms such as those utilizing additional molecular gradients and reliable feed-forward hierarchical control networks (Newman, 2003; Salazar-Ciudad et al., 2001). In work in progress, therefore, we have been considering, in the context of a hybrid continuum-discrete simulation framework, models in which the reaction-diffusion system (2.10)-(2.11) is subject to imposed gradients (representing Shh, Hox proteins, etc.) which we presume to exert a stabilizing control on the production of the system's core morphogens and extracellular matrix molecules. Because this simple reaction-diffusion system has been derived in an analytically rigorous fashion from a more complex system incorporating a large portion of the presumed core mechanism of limb development, it is reasonable to expect that simulations utilizing it will represent developmentally authentic 
phenomena within the constraints of the morphostatic assumption.

\section{Acknowledgements}

This work was supported by National Science Foundation Grant Nos. IBN-034467 to SAN and MA and EF-0526854 to SAN. In addition, B.K. was partially supported by the Polish Ministry of Science and Higher Education Grant No. 1P03A01230.

\section{Appendix A: Sufficient Conditions for Turing Instability}

Let us consider spatially homogeneous steady state $\left(c_{a}^{*}, c_{i}^{*}\right)$ of the system (3.9)-(3.10). First, notice that the second component of the homogeneous steady state can be computed from the equation:

$$
J_{a}^{1}+J_{a}\left(c_{a}\right) K\left(c_{a}\right)-J_{i}\left(c_{a}\right) K\left(c_{a}\right)=0 .
$$

Let $\mathbf{A}$ denote the Jacobian of the reaction terms in (3.9)-(3.10), taken at the equilibrium $\left(c_{a}^{*}, c_{i}^{*}\right)$. That is, denoting $F\left(c_{a}, c_{i}\right)=U\left(c_{a}\right)-k_{a} c_{a} c_{i}$ and $G\left(c_{a}, c_{i}\right)=V\left(c_{a}\right)-k_{a} c_{a} c_{i}$, we have

$$
\mathbf{A}=\left(\begin{array}{cc}
F_{, c_{a}}\left(c_{a}^{*}, c_{i}^{*}\right) & F_{, c_{i}}\left(c_{a}^{*}, c_{i}^{*}\right) \\
G_{, c_{a}}\left(c_{a}^{*}, c_{i}^{*}\right) & G_{, c_{i}}\left(c_{a}^{*}, c_{i}^{*}\right)
\end{array}\right) .
$$

We need to determine for which parameter ranges the following three conditions are satisfied (see, e.g. Murray (1993)):

1. $\operatorname{Tr} \mathbf{A}=A_{11}+A_{22}<0$.

2. $\operatorname{det} \mathbf{A}>0$

3. $A_{11}>0$.

For three or more chemicals conditions for existence of the Turing instability are more complicated (see Satnoianu et al. (2000), Satnoianu and van Driessche (2005), and also Cross (1978).)

If conditions (1),(2) and (3) are satisfied then for large enough $D_{i} / D_{a}$, the system (3.9)-(3.10) undergoes a Turing instability. In order to find the appropriate parameter ranges for our model, we first write down the specific form of these conditions in our case.

Let $J_{a}$ be given by (3.14) with $n=1$ and $k_{a}=$ const, $J_{i}=J_{i}\left(c_{a}, c_{i}\right)$. Then, at a positive constant steady state of the system (3.9)-(3.10) the following holds: 
(i) The condition $A_{11}>0$ is equivalent to

$$
\left.\frac{\alpha_{, c_{a}}}{\alpha} c_{a *}\left(J_{a}^{1}+J_{a} K\right)\right]\left(c_{a *}\right)+\left(K J_{a}\right)_{, c_{a}}\left(c_{a *}\right) c_{a *}-\left(K J_{a}\right)\left(c_{a *}\right)-J_{a}^{1}>0 .
$$

(ii) The condition $\operatorname{det} \mathbf{A}>0$ is equivalent to the condition

$$
K^{\prime}\left(J_{a}-J_{i}\right)+K\left(J_{a}^{\prime}-J_{i}^{\prime}\right)<0
$$

at $c_{a}=c_{a *}$.

(iii) The condition $\operatorname{Tr} \mathbf{A}<0$ is satisfied for large enough $k_{a}$.

These statements follow from straightforward calculations.

\section{Appendix B: Proof of the statements (i), (ii), (iii) of 3.3}

\section{B.1 Proof of (i)}

First, we we will find a solution to (A.1) lying in the region $(0, s)$.

Thus for $\delta=0$, Eq.(A.1) becomes

$$
r-w z /(1+z)+z^{2} /(1+z)^{2}=0,
$$

where $z, r$ and $w$ are defined in (3.19).

For $y=z /(1+z)$ we obtain the equation

$$
r-w y+y^{2}=0
$$

hence

$$
y=\frac{1}{2}\left(w-\sqrt{w^{2}-4 r}\right) .
$$

The fact that above we have chosen the branch with the minus sign is justified by means of Lemma 5. Indeed, one computes that the condition (A.4) is equivalent to the inequality $y<w / 2$.

Below, we will prove, however, that the condition from Lemma 5 cannot be satisfied. As we noted, to satisfy this condition we must have the inequality

$$
\left(K J_{a}\right)_{, z}(z) z-\left(K J_{a}\right)(z)>J_{a}^{1}
$$

for $z=c_{a *}$. (Note that $\alpha^{\prime}$ is negative.) By means of Remark 2 and the above denotations, this condition can be written as 


$$
y^{2} \frac{1-z}{1+z}>r
$$

To see that this condition cannot be fulfilled, let us note that for a given $w, y$ changes from $w / 2$ for $r=w^{2} / 4$ to 0 for $r=0$. Moreover, the value of $y^{2} / r$ changes from 1 for $r=w^{2} / 4$ to $r / w^{2} \searrow 0$ as $r \rightarrow 0$. This decrease is monotonic as it is straightforward to see that the derivative of $y^{2} / r$ is nonnegative. Now, we may perturb our problem by changing the value $\delta / s$ away from 0 and the condition (A.3) (which was replaced by (B.3) for $\delta=0$ ) will not be satisfied. The perturbed equation for $z$ has the form

$$
r-w z /(1+z)(z /(\delta / s))^{q} /\left[1+(z /(\delta / s))^{q}\right]-z^{2} /(1+z)^{2}=0 .
$$

Expressing $z$ by $y$ we can write it in the form:

$$
r-w y(z /(\delta / s))^{q} /\left[1+(z /(\delta / s))^{q}\right]+y^{2}=0 .
$$

where $z$ should be written within the terms of $y$. It is obvious that the above equation either has a solution (originating from the initial one), which depend continuously on the parameters or the initial solution ceases to exist. If the considered positive branch $z(\delta)$ exists (and is continuous with respect to $\delta \geq 0, y^{2}(\delta) / r$ have the same properties as before, which means that the condition (A.3) cannot be fulfilled. This completes the proof.

\section{B.2 Proof of (ii)}

We first treat the most important case $n=q=1$ and then indicate how the proof generalizes to any $n=q \geq 1$. In the case at hand, the equation (A.1) for the constant steady state takes the following form:

$$
-r+y^{2}(w-1)=0,
$$

where again $y=z /(1+z)$ and $z=c_{a} / s$. This gives $y=\sqrt{r /(w-1)}$ and hence

$$
z=\frac{\sqrt{\frac{r}{w-1}}}{1-\sqrt{\frac{r}{w-1}}} .
$$

We now need to check the condition $A_{11}>0$ in Appendix A. (See Lemma 5.) In fact, we sjow that this condition can be satisfied if $r$ is taken sufficiently small (with respect to $(w-1))$. Indeed, one computes that the condition from Lemma 5 is equivalent to the inequality

$\frac{r}{w-1}\left(\frac{1-z}{1+z}-\frac{z}{(1+z)^{2}} L K_{\max }(1+L K(z))^{-1}\right)>r\left(1+\frac{z}{(1+z)^{2}} L K_{\max }(1+L K(z))^{-1}\right)$. 
Now fix some $w$ with $1<w<2$. If we let $r \rightarrow 0$, then $z \rightarrow 0$ and $K(z) \rightarrow 0$. Thus, for small enough $r>0$, the condition (B.7) is satisfied.

It remains to be checked if the condition $\operatorname{det} A>0$ can be satisfied. (See Lemma 5.) The corresponding condition can be written as

$$
\phi(z) \phi^{\prime}(z)\left(J_{a \max }-J_{\text {imax }}\right)<0,
$$

where $\phi(z)=(z /(1+z))^{2}$. Note that $\phi(z) \phi^{\prime}(z)>0$, and so the condition (B.8) is equivalent to $J_{\text {amax }}<J_{\text {imax }}$, which in turn is equivalent to $w>1$. (Recall that $w=J_{\text {imax }} / J_{\text {amax }}$.) As we assumed that indeed $w>1$, this condtion is always satisfied.

Having thus shown the validity of the lemma for the case $n=q=1$, let us note that this simple case may be generalized to any $n=q \geq 1$. First of all, let us note that if we define

$$
y=z^{n} /\left(1+z^{n}\right),
$$

then we can still use (B.6) to calculate the positive steady state of the system. Having its positive solution $y$ we obtain

$$
z^{n}=y /(1-y), \quad z=(y /(1-y))^{1 / n} .
$$

Remark 2 Let $\phi_{n}(x, s)=(x / s)^{2 n} /\left[1+(x / s)^{n}\right]^{2}$. Then the difference $\phi^{\prime}(x, s) x-\phi(x, s)$ is equal to $y^{2}\left(2 n-1-z^{n}\right) /\left(1+z^{n}\right)$, where $z=(x / s)$.

The inequality (A.3) can be written in the form

$$
\begin{gathered}
y^{2}\left(\left(2 n-1-z^{n}\right) /\left(1+z^{n}\right)-z^{n} /\left(1+z^{n}\right)^{2} n L K_{\max } /[1+L K(z)]\right)- \\
r\left(1+z^{n} /\left(1+z^{n}\right)^{2} n L K_{\max } /[1+L K(z)]\right)>0
\end{gathered}
$$

where $K(z)=K_{\max } y$.

Finally, the inequality (B.8) changes to

$$
\phi_{n}(z) \phi_{n}^{\prime}(z)\left(J_{\text {amax }}-J_{\text {imax }}\right)<0,
$$

where we denoted $\phi_{n}(z)=\phi_{n}(x, s)$.

Based on the above remarks, we can easily repeat the above considerations.

\section{B.3 Proof of (iii)}

The equation for the constant steady state (B.6) takes the form

$$
-r+y^{2}(w \psi(z, a)-1)=0,
$$


where

$$
\psi(z, a)=\frac{a(1+z)}{1+a z}, \quad y=z /(1+z), \quad a=\frac{s}{\delta} .
$$

This is in turn equivalent to the equation

$$
z^{3}+b_{2}(a, r) z^{2}+b_{1}(a, r) z+b_{0}(a, r)=0,
$$

where the coefficients of the above polynomial are given by

$$
b_{2}(a, r)=\frac{a w-1-r(1+2 a)}{a(w-1-r)}, \quad b_{1}(a, r)=-\frac{r(a+2))}{a(w-1-r)}, \quad b_{0}(a, r)=-\frac{r}{a(w-1-r)} .
$$

Note that if $a$ and $r$ are small enough, then all the coefficients $b_{0}, b_{1}$ and $b_{2}$ are negative. Denote by $z_{1}(r), z_{2}(r)$ and $z_{3}(r)$ the three roots of (B.12) for fixed $w>1, a \ll 1$. At $r=0$, we have $z_{1}(0)=(1-a w) /(a(w-1)), z_{2}(0)=z_{3}(0)=0$. It is not hard to see that for $r>0$, the branches $z_{2}(r)$ and $z_{3}(r)$ cannot be nonnegative real numbers. Indeed, we have

$$
z_{i}^{3}=-b_{2}(r) z_{i}^{2}-b_{1}(r) z_{i}-b_{0}(r)
$$

with $-b_{2}(0)>0$ and $-b_{k}(r) \geq 0(k=0,1,2)$. One sees that for $z_{i}(r) \rightarrow 0$, one cannot have $z_{i}(r) \geq 0$.

Thus the only valid branch for small $r>0$ is $z_{1}(r)$ with $z_{1}(0)=(1-a w) /(a(w-1))$. As computed before in (B.7), the condition $A_{11}<0$ in Appendix A is equivalent to

$$
\left(\frac{z}{1+z}\right)^{2}\left(\frac{1-z}{1+z}-\frac{z}{(1+z)^{2}} L K_{\max }(1+L K(z))^{-1}\right)>r\left(1+\frac{z}{(1+z)^{2}} L K_{\max }(1+L K(z))^{-1}\right) \text {. }
$$

Note that $z_{1}(0) \rightarrow+\infty$ as $a \rightarrow 0$, and so the left hand side of the above expression is negative for small $a>0$, whereas the right hand side is always positive. It follows that we cannot have a Turing bifurcation at $r=0$ for small enough $a>0$. By continuity, this proves the claim.

\section{References}

[Alber et al. (2003)] Alber, M. S., Kiskowski, M. A., Glazier, J. A., and Jiang, Y. (2003). On cellular automaton approaches to modeling biological cells. In "Mathematical systems theory in biology, communication, and finance" (J. Rosenthal and G. D.S., Eds.), Vol. 134, pp. 1-40. Springer-Verlag, New York.

[Alber et al. (2004)] Alber, M., Kiskowski, M., Jiang, Y., and Newman, S. (2004). Biological lattice gas models. In "Dynamics and Bifurcation of Patterns in Dissipative Systems" (G. Dangelmayr and I. Oprea, Eds.), pp. 274-291. World Scientific, Singapore. 
[Alber et al. (2005a)] Alber, M., Hentschel, H.G.E., Kazmierczak, B. and Newman, S.A. (2005). Existence of solutions to a new model of biological pattern formation. Journal of Mathematical Analysis and Applications 308, 175-194.

[Alber et al. (2005b)] Alber, M., Hentschel, H.G.E., Glimm, T., Kazmierczak, B. and Newman, S.A., (2005). Stability of $n$-dimensional patterns in a generalized Turing system: implications for biological pattern formation, Nonlinearity 18, 125-138

[Brockes and Kumar (2005)] Brockes, J.P, Kumar, A., (2005). Appendage regeneration in adult vertebrates and implications for regenerative medicine. Science 310, 1919-1923.

[Chaturvedi et al. (2005)] Chaturvedi, R., Huang, C., Kazmierczak, B., Schneider, T., Izaguirre, J.A., Newman, S.A., Glazier, J.A., Alber, M. (2005). On Multiscale Approaches to 3-Dimensional Modeling of Morphogenesis, J. R. Soc. Interface 2, 237253.

[Y. Cheng and C.-W. Shu (2006)] Y. Cheng and C.-W. Shu (2006). A discontinuous Galerkin finite element method for time dependent partial differential equations with higher order derivatives. Mathematics of Computation, to appear.

[Cickovski et al. (2005)] Cickovski, T., Huang, C., Chaturvedi, R., Glimm, T., Hentschel, H. G. E., Alber, M., Glazier, J. A., Newman, S. A., and Izaguirre, J. A. (2005). A framework for three-dimensional simulation of morphogenesis. IEEE/ACM Trans. Computat. Biol. Bioinformatics 2, 273-288.

[Coates and Clack (1990)] Coates, M.I. and Clack, J.A. (1990). Polydactyly in the earliest known tetrapod limbs. Nature 347, 66-69.

[Cockburn et al. (1989a)] B. Cockburn and C.-W. Shu (1989). TVB Runge-Kutta local projection discontinuous Galerkin finite element method for conservation laws II: general framework. Mathematics of Computation 52, 411-435.

[Cockburn et al. (1989b)] B. Cockburn, S.-Y. Lin and C.-W. Shu (1989). TVB RungeKutta local projection discontinuous Galerkin finite element method for conservation laws III: one dimensional systems. Journal of Computational Physics 84, 90-113.

[Cockburn et al. (1990)] B. Cockburn, S. Hou and C.-W. Shu (1990). The Runge-Kutta local projection discontinuous Galerkin finite element method for conservation laws IV: the multidimensional case. Mathematics of Computation 54, 545-581.

[Cockburn et al. (1991)] B. Cockburn and C.-W. Shu (1991). The Runge-Kutta local projection P1-discontinuous Galerkin finite element method for scalar conservation laws. Mathematical Modelling and Numerical Analysis 25, 337-361. 
[Cockburn et al. (1998a)] B. Cockburn and C.-W. Shu (1998). The Runge-Kutta discontinuous Galerkin method for conservation laws V: multidimensional systems. Journal of Computational Physics 141, 199-224.

[Cockburn et al. (1998b)] B. Cockburn and C.-W. Shu (1998). The local discontinuous Galerkin method for time-dependent convection-diffusion systems. SIAM Journal on Numerical Analysis 35, 2440-2463.

[Crampin et al. (2002)] Crampin, E.J., Hackborn, W.W. and Maini, P.K. (2002). Pattern formation in reaction-diffusion models with nonuniform domain growth. Bull Math Biol 64, 747-69.

[Cross (1978)] Cross, G.W. (1978). Three types of matrix instability, Journal of Linear Algebra and its Applications 20, p.253-263.

[Daeschler et al. (2006)] Daeschler, E.B., Shubin, N.H. and Jenkins, F.A., Jr. (2006). A Devonian tetrapod-like fish and the evolution of the tetrapod body plan. Nature $\mathbf{4 4 0}$, $757-763$.

[De Joussineau et al. (2003)] De Joussineau, C., Soule, J., Martin, M., Anguille, C., Montcourrier, P., Alexandre, D. (2003). Delta-promoted filopodia mediate long-range lateral inhibition in Drosophila. Nature 426, 555-559.

[Dillon (2001)] Dillon, R., (2001) Mathematical modelling of vertebrate limb development, in Mathematical models in biological pattern formation, Maini, P.K. and Othmer, H. G., Springer 2001.

[Endo et al. (2004)] Endo, T., Bryant, S.V, Gardiner, D.M. (2004). A stepwise model system for limb regeneration. Dev Biol 270, 135-145.

[Entchev et al. (2000)] Entchev, E.V., Schwabedissen, A. and Gonzalez-Gaitan, M. (2000). Gradient formation of the TGF- $\beta$ homolog Dpp. Cell 103, 981-91.

[Filion and Poper (2004)] Filion, R.J. and Popel, A.S. (2004). A reaction-diffusion model of basic fibroblast growth factor interactions with cell surface receptors. Ann Biomed Eng 32, 645-63.

[Forgacs and Newman (2005)] Forgacs, G. and Newman, S.A. (2005). Biological physics of the developing embryo. Cambridge Univ. Press, Cambridge.

[Franssen et al. (2005)] Franssen, R.A., Marks, S., Wake, D., Shubin N. (2005). Limb chondrogenesis of the seepage salamander, Desmognathus aeneus (Amphibia: Plethodontidae). J. Morphol. 265, 87-101. 
[Frenz et al. (1989)] Frenz, D.A., Jaikaria, N.S. and Newman, S.A. (1989). The mechanism of precartilage mesenchymal condensation: a major role for interaction of the cell surface with the amino-terminal heparin-binding domain of fibronectin. Dev. Biol. 136, $97-103$.

[Fujimaki et al. (2006)] Fujimaki, R., Toyama, Y., Hozumi, N., Tezuka, K. (2006). Involvement of Notch signaling in initiation of prechondrogenic condensation and nodule formation in limb bud micromass cultures. J. Bone Miner. Metab. 24, 191-198.

[Gehris et al. (1997)] Gehris, A.L., Stringa, E., Spina, J., Desmond, M.E., Tuan, R.S. and Bennett, V.D. (1997). The region encoded by the alternatively spliced exon IIIA in mesenchymal fibronectin appears essential for chondrogenesis at the level of cellular condensation. Dev. Biol. 190, 191-205.

[Hartmann et al. (2006)] Hartmann, D., and Miura, T. (2006). Modelling in vitro lung branching morphogenesis during development. J Theor Biol 242, 862-72.

[Hentschel et al. (2004)] Hentschel, H.G.E., Glimm, T., Glazier, J.A., and Newman, S.A. (2004). Dynamical Mechanisms for Skeletal Pattern Formation in the Vertebrate Limb, Proc. R. Soc. B 271 1713-1722.

[Hinchliffe (2002)] Hinchliffe, J.R. (2002). Developmental basis of limb evolution. Int $J$ Dev Biol 46, 835-45.

[Izaguirre et al. (2004)] Izaguirre, J.A., Chaturvedi, R., Huang, C., Cickovski, T., Coffland, J., Thomas, G., Forgacs, G., Alber, M., Hentschel, G., Newman, S. A., Glazier, J.A. (2004). CompuCell, a multi-model framework for simulation of morphogenesis, Bioinformatics 20, 1129-1137.

[Johnson (1987)] C. Johnson (1987). Numerical solution of partial differential equations by the finite element method. Cambridge University Press, Cambridge-New York-New Rochelle-Melbourne-Sydney.

[Lander (2007)] Lander, A.D. (2007). Morpheus unbound: reimagining the morphogen gradient. Cell 128, 245-56.

[Lander et al. (2002)] Lander, A.D., Nie, Q. and Wan, F.Y. (2002). Do morphogen gradients arise by diffusion? Dev Cell 2, 785-96.

[Leonard et al. (1991)] Leonard, C.M., Fuld, H.M., Frenz, D.A., Downie, S.A., Massagu J. and Newman, S.A. (1991). Role of transforming growth factor- $\beta$ in chondrogenic pattern formation in the embryonic limb: stimulation of mesenchymal condensation and fibronectin gene expression by exogenous TGF- $\beta$ and evidence for endogenous TGF- $\beta$-like activity. Dev. Biol. 145, 99-109. 
[Litingtung (2002)] Litingtung, Y., Dahn, R. D., Li, Y., Fallon, J. F., and Chiang, C. (2002). Shh and Gli3 are dispensable for limb skeleton formation but regulate digit number and identity. Nature 418, 979-83.

[Lyons and Harrison (1992)] Lyons, M. J. and Harrison, L. G. (1992). Stripe selection: an intrinsic property of some pattern-forming models with nonlinear dynamics. Dev. Dyn. 195, 201-215.

[Maree and Hogeweg (2001)] Maree, A. F., and Hogeweg, P. (2001). How amoeboids selforganize into a fruiting body: multicellular coordination in Dictyostelium discoideum. Proc Natl Acad Sci U S A 98, 3879-83.

[Martin (1998)] Martin, G.R. (1998). The roles of FGFs in the early development of vertebrate limbs. Genes Dev 12, 1571-86.

[Merks and Glazier (2005)] Merks, R. M. H., and Glazier, J. A. (2005). A cell-centered approach to developmental biology. Physica A 352, 113-130.

[Merkin and Sleeman (2005)] Merkin, J.H. and Sleeman, B.D. (2005). On the spread of morphogens. J. Math. Biol. 51, 1-17.

[Miura and Shiota (2000a)] Miura, T. and Shiota, K. (2000). TGF- $\beta 2$ acts as an "activator" molecule in reaction-diffusion model and is involved in cell sorting phenomenon in mouse limb micromass culture. Dev. Dyn. 217, 241-249.

[Miura and Shiota (2000b)] Miura, T., Shiota, K. (2000). Extracellular matrix environment influences chondrogenic pattern formation in limb bud micromass culture: Experimental verification of theoretical models. Anat. Rec. 258, 100-107.

[Miura and Shiota (2002)] Miura, T., and Shiota, K. (2002). Depletion of FGF acts as a lateral inhibitory factor in lung branching morphogenesis in vitro. Mech. Dev. 116, $29-38$.

[Miura and Maini (2004)] Miura, T. and Maini, P.K. (2004). Speed of pattern appearance in reaction-diffusion models: implications in the pattern formation of limb bud mesenchyme cells. Bull. Math. Biol. 66, 627-49.

[Miura et al. (2006)] Miura T., Shiota K., Morriss-Kay G., Maini P.K. (2006) Mixedmode pattern in Doublefoot mutant mouse limb-Turing reaction-diffusion model on a growing domain during limb development. $J$ Theor Biol. 240, 562-73.

[Moftah et al. (2002)] Moftah, M.Z., Downie, S.A., Bronstein, N.B., Mezentseva, N., Pu, J., Maher, P.A. and Newman, S.A. (2002). Ectodermal FGFs induce perinodular 
inhibition of limb chondrogenesis in vitro and in vivo via FGF receptor 2. Dev Biol 249, 270-82.

[Murray (1993)] Murray, J.D., Mathematical Biology, Springer, Berlin, 2nd edition, 1993.

[Nelson et al. (2006)] Nelson, C. M., Vanduijn, M. M., Inman, J. L., Fletcher, D. A., and Bissell, M. J. (2006). Tissue geometry determines sites of mammary branching morphogenesis in organotypic cultures. Science 314, 298-300.

[Newman and Frisch (1979)] Newman, S.A., and Frisch, H. (1979). Dynamics of skeletal pattern formation in developing chick limb, Science 205, 662-668.

[Newman (1988)] Newman, S.A. (1988). Lineage and pattern in the developing vertebrate limb. Trends Genet. 4, 329-332.

[Newman (2003)] Newman, S.A. (2003). From physics to development: the evolution of morphogenetic mechanisms. In: Müller GB, Newman SA, editors. Origination of Organismal Form: Beyond the Gene in Developmental and Evolutionary Biology. Cambridge, MA.: MIT Press. p 221-239.

[Newman and Müller (2005)] Newman, S.A. and Müller, G.B. (2005). Origination and innovation in the vertebrate limb skeleton: an epigenetic perspective. $J$ Exp Zoolog B Mol Dev Evol 304, 593-609.

[Newman and Forgacs (2005)] Newman, S.A. and Forgacs, G. (2005). Complexity and self-organization in biological development and evolution. In Bonchev, D. and Rouvray, D.H. (eds.), Complexity in chemistry, biology and ecology, Springer, Berlin, pp. 49-95.

[Nijhout (2003)] Nijhout, H.F. (2003). Gradients, diffusion and genes in pattern formation. In Müller, G.B. and Newman, S.A. (eds.), Origination of Organismal Form: Beyond the Gene in Developmental and Evolutionary Biology., MIT Press, Cambridge, MA., pp. $165-181$.

[Pao (1992)] Pao, C.V. (1992). Nonlinear Parabolic and Elliptic Equations Plenum Press, New York.

[Salazar-Ciudad et al. (2001)] Salazar-Ciudad, I., Newman, S.A. and Solé, R. (2001). Phenotypic and dynamical transitions in model genetic networks. I. Emergence of patterns and genotype-phenotype relationships. Evolution 85 Development 3, 84-94.

[Salazar-Ciudad et al. (2003)] Salazar-Ciudad, I., Jernvall, J. and Newman, S.A. (2003). Mechanisms of pattern formation in development and evolution. Development 130, 2027-37. 
[Salazar-Ciudad (2006)] Salazar-Ciudad, I. (2006). On the origins of morphological disparity and its diverse developmental bases. Bioessays 28, 1112-22.

[Satnoianu et al. (2000)] Satnoianu, R. A., Menzinger, M., and Maini, P.K. (2000). Turing instabilities in general systems, J. Math. Biol. 41, 493-512

[Satnoianu and van den Driessche (2005)] Satnoianu, R.A. and van den Driessche, P. (2005). Some remarks on matrix stability with application to Turing instability, Journal of Linear Algebra and its Applications 398, 69-74.

[Shubin et al. (2003)] Shubin, N.H., Daeschler, E.B. and Jenkins, F.A., Jr. (2006). The pectoral fin of Tiktaalik roseae and the origin of the tetrapod limb. Nature 440, $764-771$.

[Stark (2003)] Stark, R.J. and Searls, R.L. (1973). A description of chick wing bud development and a model of limb morphogenesis. Dev Biol. 33, 138-153.

[Tickle (2003)] Tickle, C. (2003). Patterning systems-from one end of the limb to the other. Dev Cell 4, 449-58.

[Turing (1952)] Turing, A.M. (1952). The chemical basis of morphogenesis. Phil. Trans. R. Soc. Lond. B 237, 37-72.

[Waddington (1942)] Waddington, C.H. (1942). Canalization of development and the inheritance of acquired characters. Nature 150, 563-565.

[Wagner (2005)] Wagner, A. (2005). Robustness and Evolvability in Living Systems. Princeton, Princeton University Press

[Wei et al. (2006)] Wei, G., Schubiger, G., Harder, F., and Muller, A. M. (2000). Stem cell plasticity in mammals and transdetermination in Drosophila: common themes? Stem Cells 18, 409-14.

[Williams et al. (2004)] Williams, P.H., Hagemann, A., Gonzalez-Gaitan, M. and Smith, J.C. (2004). Visualizing long-range movement of the morphogen Xnr2 in the Xenopus embryo. Curr Biol 14, 1916-23.

[Zhu and Scott (2004)] Zhu, A.J. and Scott, M.P. (2004). Incredible journey: how do developmental signals travel through tissue? Genes Dev 18, 2985-2997.

[Zykov and Engel (2004)] Zykov, V. and Engel, H. (2004). Dynamics of spiral waves under global feedback in excitable domains of different shapes. Phys. Rev. E Stat. Nonlin. Soft Matter Phys. 70: 016201. 
Fig. 1: Cartoon representing semi-transparent view of chondrogenesis in the chick wing bud between 4 and 7 days of development. Light gray represents precartilage (sites of R2 and R2' cells) and black represents definitive cartilage (sites of R3 cells). Adapted, with modifications from Forgacs and Newman (2005).

Fig. 2: A graph illustrating the effect of the parameter $\delta / s$ on the Turing instability. We have numerically solved the system (3.9)-(3.10) in the one-dimensional interval $[0,1]$ with no-flux boundary conditions for different values of the parameter $\delta$. The computations were run for each parameter $\delta$ until a steady state was reached. The parameter values were chosen such that the most unstable wavelength was $k=6$, corresponding to three spatial concentration peaks. The graph shows these steady states, parameterized by the quotient $\delta / s$. Thus each line represents a steady state of the system, corresponding to a specific value of the parameter $\delta / s$.

The Turing instability holds for $\delta / s$ between approximately 1.0 and 1.3 . Note that while the above graph represents only a specific example, it is shown in the paper that this qualitative behavior holds in general, in the sense that the Turing instability is impossible for $\delta / s>>1$ and $\delta / s<<1$. The specific parameters for this computations were as follows: $J_{a, \max }=6.0 \gamma, J_{i, \max }=8.0 \gamma, s=4.0, k_{a}=\gamma, D_{a}=1, D_{i}=100.3, J_{a}^{1} \alpha=0.05 \gamma$, $\beta\left(c_{a}\right)=0.693473 c_{a} /\left(c_{a}+2.66294\right), R_{e q}=2.0$ with $\gamma=8900$.

Figure 3A: An illustration of the Turing space of the system (3.9)-(3.10). All parameters except $J_{\text {imax }}$ and $\delta$ were kept constant: $J_{a, \max }=6.0 \gamma, s=4.0, k_{a}=\gamma, J_{a}^{1}=0.05 \gamma$, $K_{1}\left(c_{a}\right)=4 c_{a} / 8.6+c_{a}, K_{2}=0.9 / 11.9, R_{e q}=2.0$ with $\gamma=8900$.

Figure 3B: An illustration of the Turing space of the system (3.9)-(3.10). Here, all parameters except the cell density $R_{e q}$ and $\delta$ were kept constant. The values of parameters for this simulation were chosen as in Figure 3A, except for $J_{i, \max }=8.0$. 
Fig. 4A: Simulations of the active zone with an irregular shape. Contour plots of the steady state of the concentration of the activator. A triangular mesh is used to fit the irregular boundary of the domain. The vertical length of the domain is roughly 0.65 , and the horizontal length is 0.15 . Parameter values in the system: $D_{a}=1, D_{i}=50.3, J_{a}^{1} \alpha=$ $0.05 \gamma, J_{\text {amax }}=6.0 \gamma, J_{\text {imax }}=8.0 \gamma, k_{a}=\gamma, \beta_{1}=0.693473, \beta_{2}=2.66294, R_{e q}=2.0, n=$ $q=2, \gamma=8900, s=4.0, \delta=4.8$.

Fig. 4B: Simulations of active zones with changing sizes in the horizontal direction, and the vertical size fixed at 1 . Contour plots of the steady states of concentrations of the activator for different domain size. Triangular meshes are used. Parameter values in the system are the same as those in Fig. 4A. 


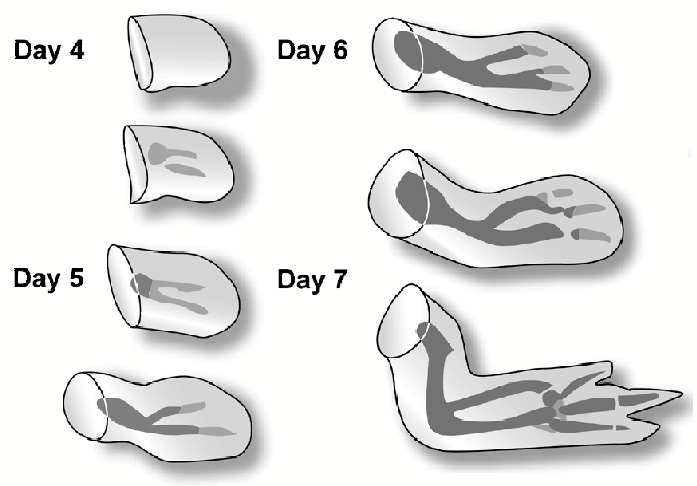

Fig. 1

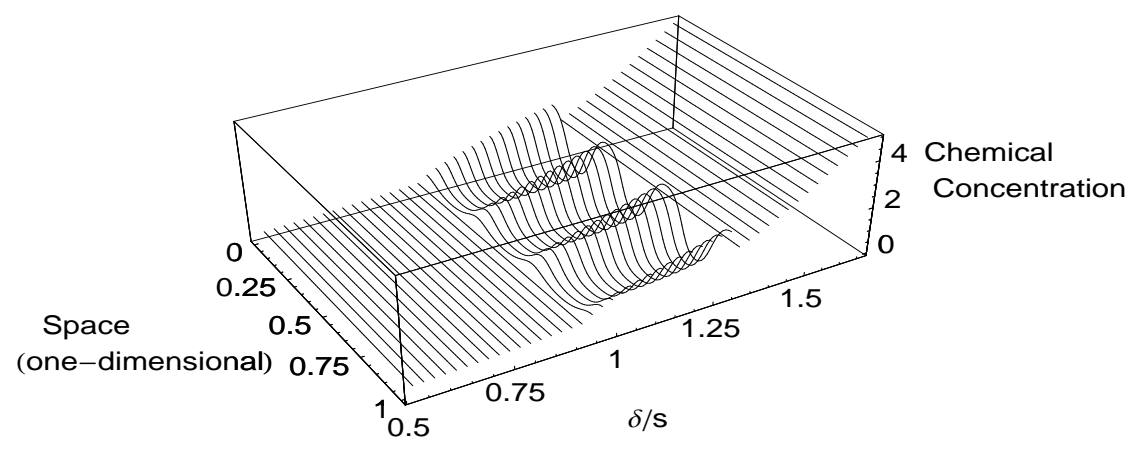

Fig. 2 


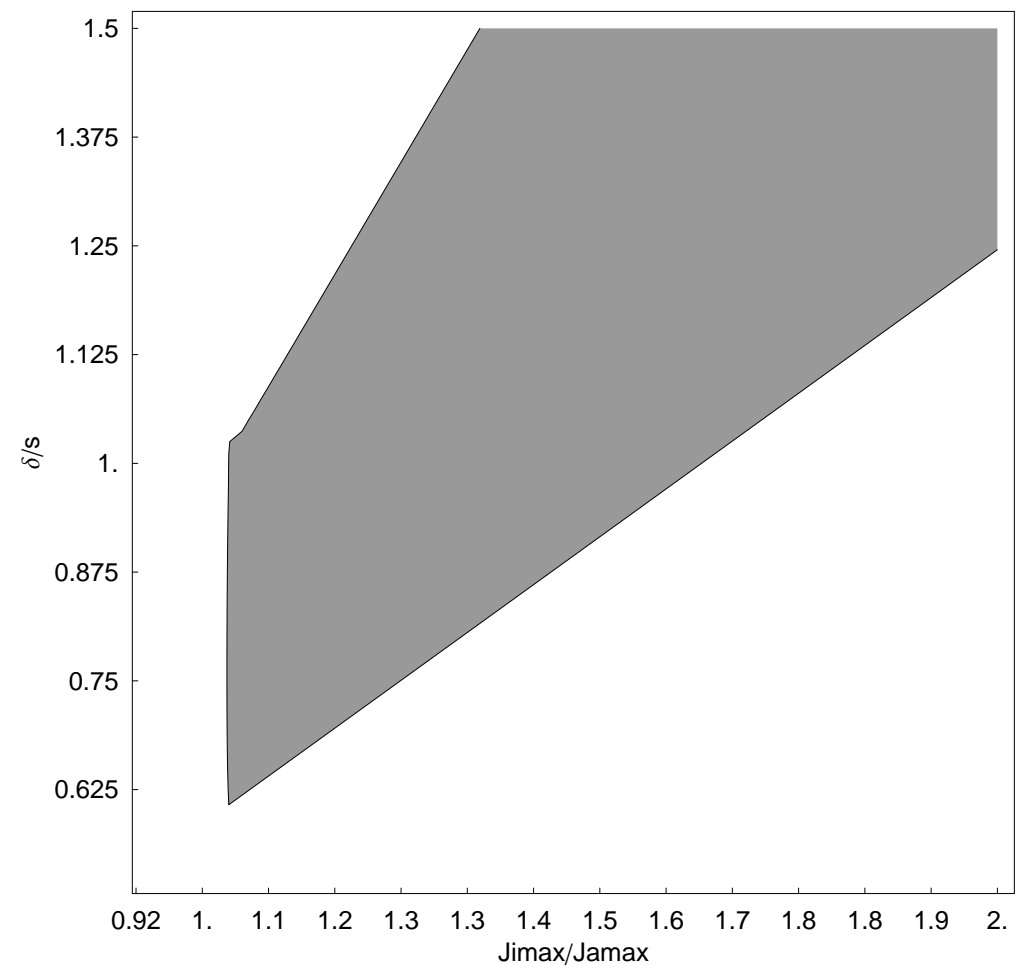

Fig. 3A 


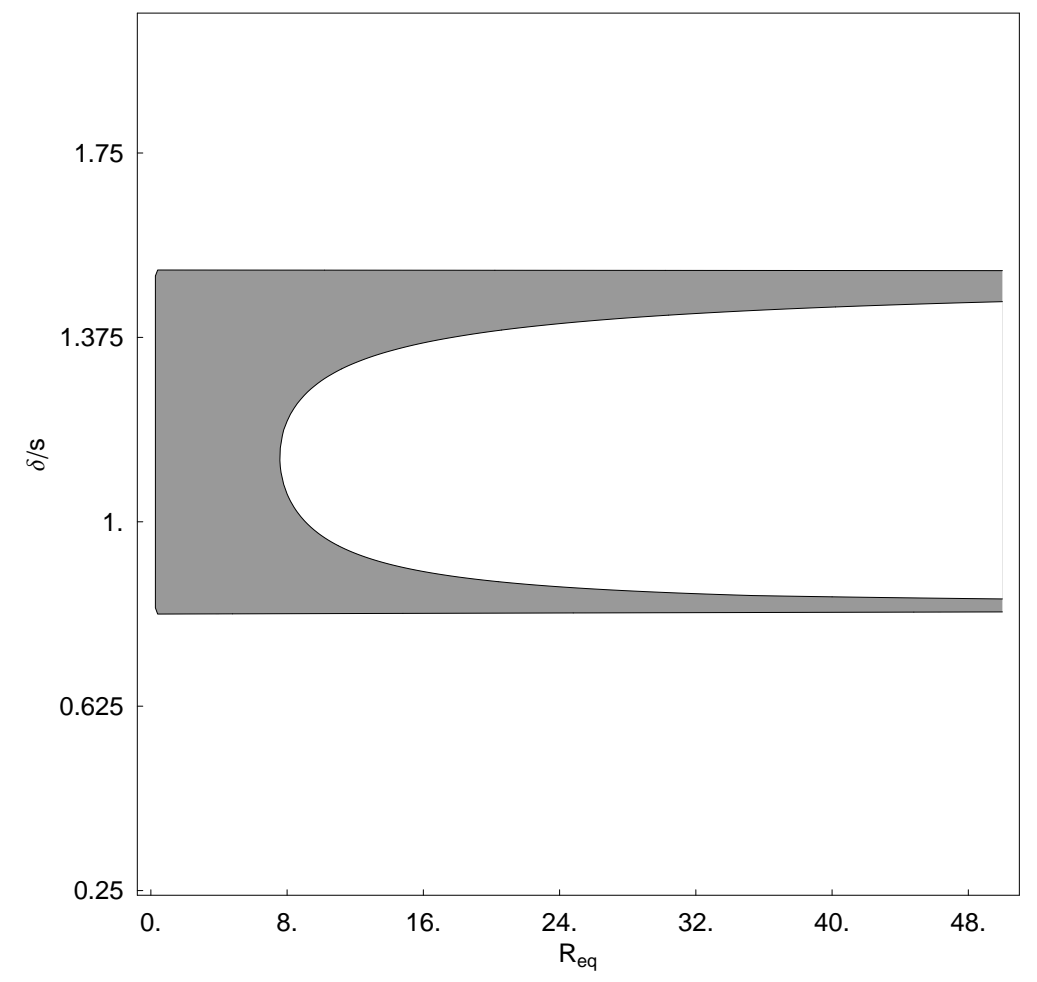

Fig. 3B 


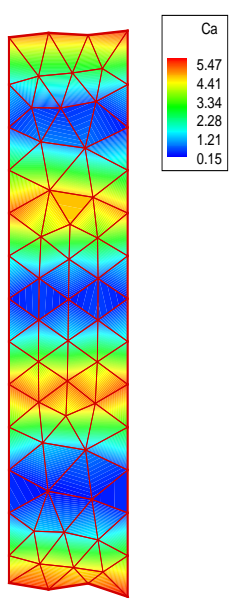

Fig. 4A
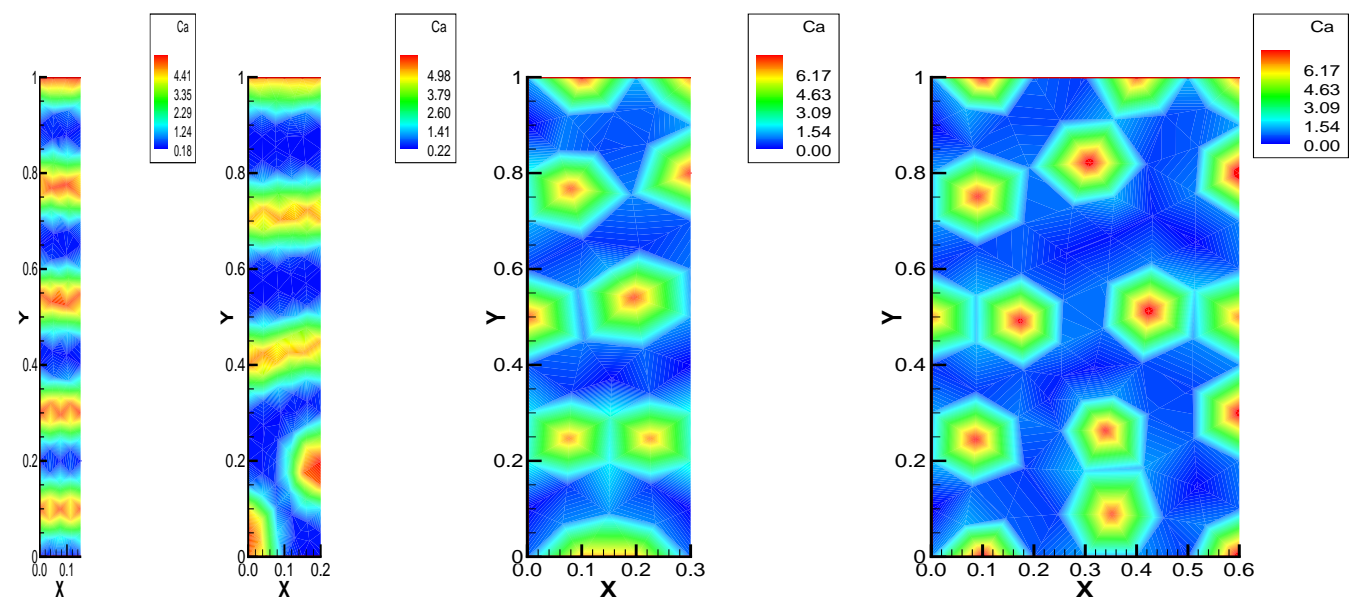

Fig. 4B 